Document downloaded from:

http://hdl.handle.net/10251/51847

This paper must be cited as:

Vilaplana Cerda, RI.; Luna Molina, R. (2011). The shape influence on the overall single scattering properties of a sample in random orientation. Journal of Quantitative Spectroscopy and Radiative Transfer. 112(11):1838-1847. doi:10.1016/j.jqsrt.2011.01.006.

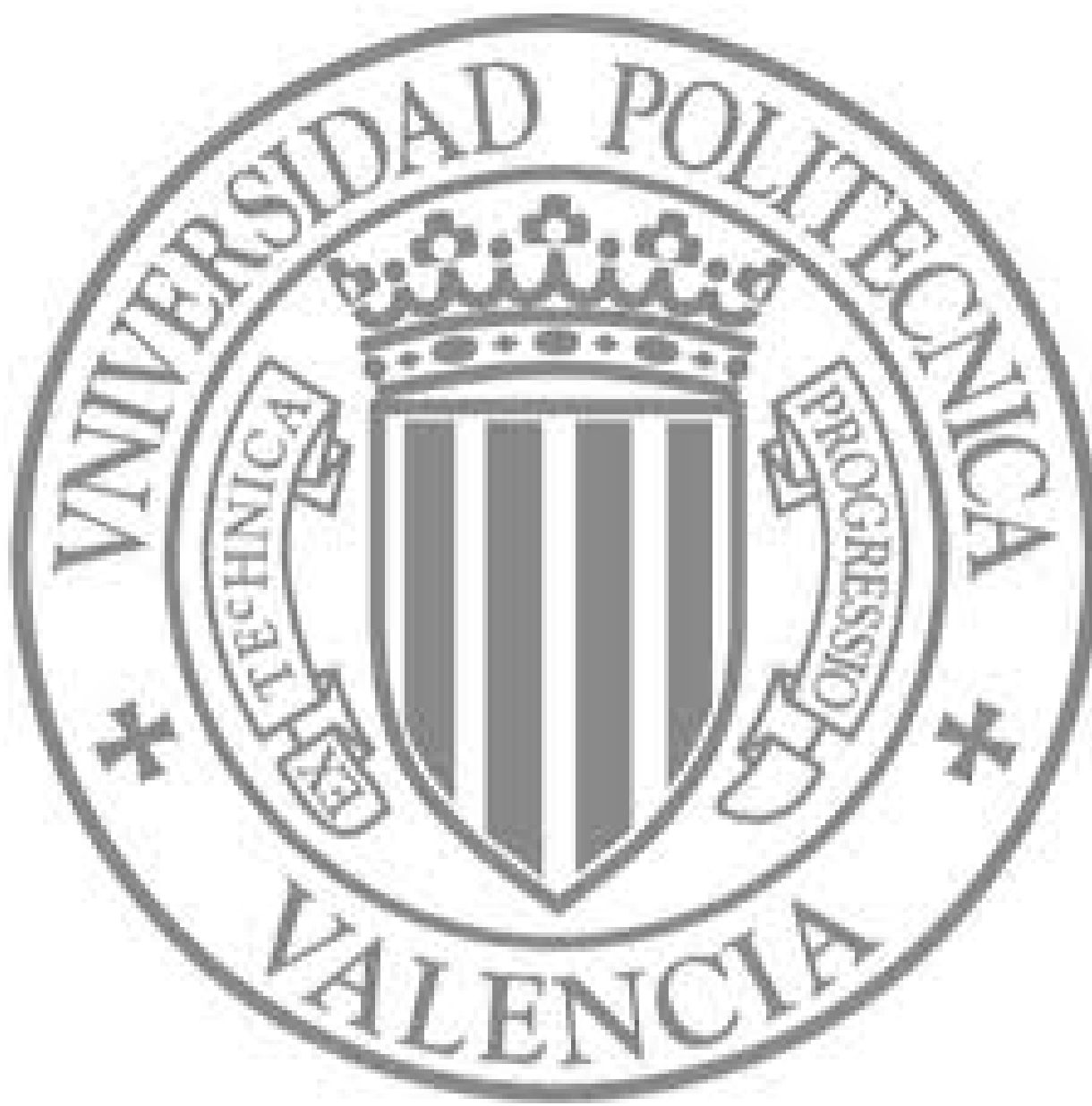

The final publication is available at

http://dx.doi.org/10.1016/j.jqsrt.2011.01.006

Copyright Elsevier 


\title{
The shape influence on the overall single scattering properties of a sample in random orientation
}

\author{
R.Vilaplana ${ }^{\mathrm{a}, *}$, R.Luna ${ }^{\mathrm{a}}$, D.Guirado ${ }^{\mathrm{b}}$, \\ ${ }^{a}$ Centro de Tecnologías Físicas, Universidad Politécnica de Valencia, Escuela Politécnica \\ Superior de Alcoy, Alcoy, Alicante, Spain 03801 \\ ${ }^{b}$ Instituto Astrofísico de Andalucía, Granada, Spain
}

\section{Abstract}

The particle shape influences the curves of the scattering efficiency factor $\left(\mathrm{Q}_{\text {sca }}\right)$ as a function of the size parameter $(\mathrm{X})$, and consequently on the overall single scattering properties of a sample of particles in random orientation. In order to show how the influence of the particle shape works, a model consisting of aggregates of different numbers of spheres has been used to fit laboratory measurements of fly ashes. The results for other shapes, such as rectangular prisms with different axial proportions, particles made of joined cubes, and particles with different fluffiness, are also shown. From all these calculations, it is concluded that the size averaged scattering matrix element resembles Rayleigh features, for the size distribution stopping at $1.0 \mu \mathrm{m}$, when either the number of spheres or cubes of the aggregates is increased, the shape becomes flatter or the fluffiness degree is increased.

Keywords: Single scattering, Scattering efficiency, nonspherical particles

\footnotetext{
${ }^{*}$ Corresponding author

Email addresses: rovilap@fis.upv.es (R.Vilaplana), ralunam@fis.upv.es (R.Luna), dani@iaa.es (D.Guirado) 


\section{Introduction}

The measurements carried out in the scattering laboratories [1] and the astronomical observations [2] provide with information on the overall scattering properties of a sample formed by small particles in random orientation. Single scattering properties of a distribution of particles in random orientation depend on properties of the grains, such as refractive index, size, shape, and the degree of fluffiness of the particles. Individually identifying or "untangling" the way in which each parameter is affecting the overall scattering properties is difficult since these parameters have a collective influence on the scattering pattern. In order to better understand how the single scattering properties observed are affected by these single parameters, a lot of research has been carried out [3-7]; however, some questions remain open. The goal of this study is to show how the particles shape influences the single scattering matrix elements of a sample of small particles in random orientation. In order to do this, we show the curves of $\mathrm{Q}_{s c a}$ vs. X for different shapes and the overall single scattering properties of several samples of particles in random orientation. Although, our main goal is not to exactly fit the laboratory measurements, we present an attempt to fit a set of scattering laboratory measurements [1] by modelling it as a distribution of different aggregates of spheres. This comparison becomes a good example to point out how $\mathrm{Q}_{\text {sca }}$ vs. X, and consequently the overall single scattering properties, is affected by the particles shape. The results for other particle shapes are also showed to clarify the shape influence on the overall single scattering properties. 


\section{Model}

The scattering matrix of a sample of particles of different sizes and the same shape is calculated as in Eq. (1):

$$
F_{i j}(\lambda, \theta)=\int_{r 1}^{r 2} F^{i j}(\lambda, \theta, r) n(r) d r
$$

where $n(r)$ is the size distribution as a function of the radius, $r_{1}$ and $r_{2}$ correspond to the smallest and largest particles in the distribution respectively, and $F^{i j}(\lambda, \theta, r)$ is one of the elements of the scattering matrix for a single particle of radius $\mathrm{r}$, at a certain wavelength $\lambda$ for a scattering angle $\theta$.

We have used the DDA (Discrete Dipole Approximation) for all calculations [8] because it has the potential to reproduce any particle shape, although it is not suitable for performing calculations for particles much larger than the wavelength of the incident light, on the current computers, in a reasonable time (days). For all our calculations, the size distribution was chosen a power law with an index of -1.8 , and 35 equally spaced radii between 0.1 and $1.0 \mu \mathrm{m}$. The calculations were averaged over 2000 orientations, to mimic the random orientation, and the number of dipoles was chosen so that the accuracy condition $|\operatorname{mkd}|<0.5$ was fulfilled [9].

\section{Calculations for aggregate of spheres compared to measure- ments}

The first shapes considered were aggregates of spheres. In this case, we have compared our calculations with the single scattering laboratory measurements of fly ashes [1]. The sample used in these measurements resembles 
aggregates of spheres. In Fig. 1, we show an image of the eight aggregates considered in our calculations. These aggregates are made of 5, 7, 7 in a line, 9, 14, 19, 25 and 36 spheres, so the four on the top are made of a smaller number of spheres than those on the botton. The value of the refractive index was chosen $1.5+0.001 \mathrm{i}$ and the wavelength $0.633 \mu \mathrm{m}$, as given in [1].

In Fig. 2, we show the comparison of the laboratory measurements with our results, size averaged, for the eight aggregates of spheres showed in the Fig. 1, plus a single sphere. In Fig. 2, we can see the overlapped images of the size averaged results for each of the aggregates of spheres (blue and red lines) and for a single sphere (dashed-dot-dot black line). It comes out from Fig. 2 that the contribution of the aggregates of less number of spheres $(\leq 9)$ is necessary to approach the laboratory measurements (see in Fig. 2 the blue and red lines). We can also notice a tendency to resemble the Rayleigh features of the scattering matrix elements as functions of the scattering angle when the aggregates are made of more spheres (see in Fig. 2 the dashed-dot red lines). On the other hand, we note that the real size distribution, as given in the reference [1], has constituents with $\mathrm{X}$ larger than 10. In Fig. 3 , we show comparison of the laboratory measurements with our results size and shape averaged considering the eight aggregates of spheres showed in Fig. 1 plus a single sphere equally weighted. Without the aggregates with less number of spheres (see blue lines in Fig. 2) the size and shape average can not even approach the measurements.

In Fig. 3, we see a not perfect fit of the results of DDA to the measurements, the calculations stopping at $\mathrm{r}_{2}=1.0 \mu \mathrm{m}$. In particular, the deviation of the calculated values from the measurements points to a Rayleigh-like be- 
haviour. From Fig. 2, we infer that the more spheres the aggregates are made of, the more the calculated values resemble Rayleigh features of the scattering matrix elements as functions of the scattering angle. This is suggesting us an explanation for the unperfected fitting: when aggregates are made of a large number of spheres, the curve of $\mathrm{Q}_{\text {sca }}$ as a function of $\mathrm{X}$ changes so that we are skipping some of its main features by cutting our size distribution at $\mathrm{r}_{2}=1.0 \mu \mathrm{m}$. In order to prove this, we present on Fig. 4 the $\mathrm{Q}_{\text {sca }}$ curves for the four aggregates with a number of spheres $\leq 9$ till $\mathrm{X}=10$, along with the $\mathrm{Q}_{\text {sca }}$ curve of the single sphere, calculated till $\mathrm{X}=15$. A progressive displacement to the right and rising of the $\mathrm{Q}_{\text {sca }}$ curves is observed when the number of spheres of the aggregates is increased. Due to this displacement, some of the features of $\mathrm{Q}_{\text {sca }}$ that correspond to $\mathrm{r}>1.0 \mu \mathrm{m}$ are lost in our calculations, and this effect becomes more important as the number of spheres of the aggregates increases. The result is a Rayleigh-like behaviour, because only the first oscillation of the curve of $\mathrm{Q}_{s c a}$ is been considered in the size distribution.

\section{Calculations for rectangular prisms}

The next shapes considered were rectangular prisms with different axial proportions namely 5:5:5, 5:4:4, 5:3:3, 5:2:2, 5:1:1, 5:4:1, 5:3:1, 5:2:1, 5:5:1, $5: 5: 2,5: 5: 3$, and 5:5:4. The values of the refractive index and the wavelength for these calculations were $1.62+0.09 \mathrm{i}$ and $0.6 \mu \mathrm{m}$, respectively. In Fig. 5, we show four images, which show the overlapped curves of the $\mathrm{Q}_{\text {sca }}$ vs. X, obtained for different combinations of these prisms compared with the case of a single sphere. Image a) of Fig. 5 shows the results for the prisms with the 
extreme axial proportions 5:5:5, 5:1:1 and 5:5:1. A progressive displacement to the right and rising of the $\mathrm{Q}_{s c a}$ values compared with the curve of a sphere is observed. The closest result to the sphere is for the cube and the highest displacement and rising is for the prism with axial proportion 5:5:1. The other three images in Fig.5 show the results when varying the axial proportions. Image b) of Fig. 5 shows the results for the transition between the axial proportion from 5:1:1 to 5:5:5, image c) is the transition from 5:5:5 to 5:5:1 and image d) from 5:5:1 to 5:1:1. All these images show how is affected $\mathrm{Q}_{s c a}$ vs. X depends on the axial proportion of the prisms. The flattest shapes (5:5:1, 5:4:1 and 5:3:1) give the largest displacements and risings.

In previous paper, we have compared the size-averaged scattering matrix elements as functions of the scattering angle for some rectangular prisms with axial proportions (see Fig.1 in reference [6]). The size-averaged scattering matrix elements showed in this figure shows a Rayleigh-like behaviour for the flattest rectangular prisms (5:5:1, 5:3:1 and 5:4:1). The reason is that only the first oscillations of the $\mathrm{Q}_{\text {sca }}$ curves are considerated in the size-average of the scattering matrix elements (see the values of $\mathrm{Q}_{s c a}$ curves marked with arrows on the image a) and the values of $\mathrm{Q}_{\text {sca }}$ for the axial proportions 5:5:1, 5:3:1 and 5:4:1 in image c) of Fig. 5. A similar result with the flattest shapes was obtained in other studies with platelike and needlelike particles [10] and with spheroids of different axis ratios [11]. 


\section{Calculations for aggregate of cubes}

Other shapes considered were made by joined cubes. In Fig. 6, we show an image with these shapes and the labels used. The shape called Test-h has a hole in the center so then only six cubes forms it. The B9 and B3 have the largest number of cubes, the B4 is the flattest one and the B1 and B7 differ only by a less cube in the bottom right corner of the B7. The values of the refractive index and the wavelength were again $1.62+0.09 \mathrm{i}$ and $0.6 \mu \mathrm{m}$, respectively.

In Fig. 7, we show four plots with the overlapped curves of $\mathrm{Q}_{s c a}$ vs. X for different combinations of the shapes showed in Fig. 6, compared to the result for a single sphere. Image a) of Fig. 7 show the $\mathrm{Q}_{\text {sca }}$ vs. X curves for all the shapes in Fig. 6. The highest displacement and rise of the first maximum of the $\mathrm{Q}_{\text {sca }}$ curves is observed for the particle B3 (one of the shapes made of the largest number of cubes). The effect on the $\mathrm{Q}_{\text {sca }}$ curves of increasing the number of cubes is showed more clearly in image b) of Fig. 7 for shapes B8, B2, B6, B3 and B9.The flattest shapes B4 and B5 also show high values and displacement of the first maximum of $\mathrm{Q}_{\text {sca }}$ although the number of cubes is smaller than in shapes B1 and B7. This is showed in image c) of Fig. 7. Finally, image d)of Fig. 7 shows the differences between the $\mathrm{Q}_{\text {sca }}$ values for a single the cube, Test, Test-h and B1 particles. Surprisingly, the shape B9 which is made of 100 cubes gives a low value of the first maximum however as we will show immediately after, this shape also is strongly influencing the ripples over the $\mathrm{Q}_{\text {sca }}$ curves which seems to be totally different depending of the shape.

In previous papers, we have compared the size-averaged scattering matrix 
elements as functions of the scattering angle for some of these shapes with the same values of the refractive index and wavelength (see Fig. 5 in reference [5] and Fig. 7 in reference [12]). The size-averaged scattering matrix elements showed in these figures seems to have values quite close to each other. However, in Fig. 8, we show the size-averaged scattering matrix elements and the corresponding $\mathrm{Q}_{\text {sca }}$ vs. X curves for shapes B8, B3 and B9, which have an increasing number of cubes. This figure shows again that, the more cubes the particles are made of, the more the calculated values resemble Rayleigh features of the scattering matrix elements as functions of the scattering angle. This effect can be clearly observed in previous calculations with a shape of made of 256 cubes and an equal-size configuration but having spherical monomers instead of cubes [6].In Fig.8 we have also marked with an arrows two pairs of points on $\mathrm{Q}_{s c a}$ curves in which the high-frequency ripples observed are in a equivalent state. In other words, due to the influence of the shape on the the low-frequency maxima and minima and on the high-frequency ripples some of the features of $\mathrm{Q}_{\text {sca }}$ curves that correspond to $\mathrm{r}>1.0 \mu \mathrm{m}$ are lost, becoming this effect more important as the number of cubes of the aggregates increases. The result is a Rayleigh-like behaviour, because only the first oscillation of the curve of $\mathrm{Q}_{s c a}$ is been considered in the size distribution.

\section{Calculations for fluffy particles}

The last particles considered have a fluffiness degree generated by uniformly randomly removing dipoles in different percentages. The particle refractive index and the wavelength were again $1.62+0.09 \mathrm{i}$ and $0.6 \mu \mathrm{m}$, re- 
spectively. In Fig. 9, we show the $\mathrm{Q}_{s c a}$ vs. X curves and the size-averaged scattering matrix elements as functions of the scattering angle (excluding $\left.\mathrm{F}_{44} / \mathrm{F}_{11}\right)$ for a compact sphere and this shape with fluffiness degrees of $15 \%$, $25 \%$ and $50 \%$. A displacement to the right and rising of the first maximum of $\mathrm{Q}_{\text {sca }}$ is observed as the fluffiness degree increases; which implies that the fluffier the sphere is, the more the scattering matrix elements as functions of the scattering angle resemble Rayleigh features.

Our simple way to generate the porosity degree in the particles allow as to obtain the effective refractive index by using the mixing rules of Effective Medium Approximation [13], considering the inclusion refractive index as $1.0+0.0 \mathrm{i}$. The result is that the real and imaginary part of the effective refractive index decrease simultaneously and progressively as the fluffiness degree is increased. On the other hand, in chapter 9 of reference [14] we can see the effect of varying the real and imaginary part of the refractive index on the scattering efficiencies curves for a sphere. A displacement to higher values of $\mathrm{X}$ of all the maxima of the $\mathrm{Q}_{s c a}$ curves is produced by a decrement of the real part of the refractive index; however, a decrease of the imaginary part of the refractive index produces a rise of $\mathrm{Q}_{s c a}$ maxima without changing their positions. Consequently, the displacement to the right and a rise of the $\mathrm{Q}_{\text {sca }}$ values we observed in our calculations with fluffy sphere could be understood in these terms.

To check what it happens with fluffy shapes different of a sphere, in Fig. 10 we show $\mathrm{Q}_{\text {sca }}$ vs. X curves and the corresponding size-averaged scattering matrix elements as functions of the scattering angle (excluding the $\mathrm{F}_{44} / \mathrm{F}_{11}$ ) for shapes Test, Test-h and Test with different fluffiness degrees $(14 \%, 25 \%$ 
and $50 \%$ ). The percentage of $14 \%$ corresponds to the quantity of matter eliminated in the center of the shape Test-h. It is again observed that the fluffier the shape is, the displacement and rise of the first maximum of $\mathrm{Q}_{s c a}$ become, and consequently, the scattering matrix elements as functions of the scattering angle more resemble Rayleigh features.

\section{Calculations for an equal size-configuration of cubes and spheres}

Finally, we have carried out calculations with an equal size-configuration of cubes and spheres for a very high refractive index. In Fig. 11, we show the $\mathrm{Q}_{\text {sca }}$ vs. X curve and the corresponding scattering matrix elements as functions of the scattering angle (excluding the $\mathrm{F}_{44} / \mathrm{F}_{11}$ ) for the shape $\mathrm{B} 3$ of Fig. 6, which is made of 40 cubes and an equal-size configuration but having spherical monomers instead of cubes; compared with a single sphere for a refractive index of $2.0+0.4 \mathrm{i}$. Although the size distribution stops at $1.0 \mu \mathrm{m}$, a tendency to reach the geometric optic regime is observed for the two shapes (equal-size configuration of cubes and spheres). In Fig. 11, the maximum of the lineal polarization increases, does not show negative branch, and is displaced to a scattering angle smaller than $90^{\circ}$.

\section{Discussion and conclusions}

We have carried out calculations with different shapes to shed light on how the particle shape influences the single scattering matrix elements of a sample of small particles in random orientation, concluding that the scattering efficiency is an essential parameter to understand the influence of the shape. 
The $\mathrm{Q}_{\text {sca }}$ vs. $\mathrm{X}$ curve of a sphere is characterized by a succession of major low-frequency maxima and minima with superimposed high-frequency ripples. The low-frequency maxima and minima have been traditionally explained as the "interference structure" and the high-frequency ripples as a consequence of the total reflection of the ray inside of the particle [15]. Taking into account these explanations is clear that not only the refractive index of the particles will have a strong effect on the $\mathrm{Q}_{s c a}$ vs. X curves but also the shape. One of the main conclusions, we have reached with our calculations is that fixed all the parameters of the model, the shape is influencing on the $\mathrm{Q}_{s c a}$ vs. X curve in such a way that the size-averaged scattering matrix elements resemble Rayleigh features in three cases: a) the number of spheres or cubes is increased in the particle, b) the flatter the particle is, c) the fluffiness degree of the particles is increased. In other words, in the three cases mentioned, a displacement to the right and rising of the first low-frequency maximum is observed as a consequence of stopping the size distributions at $\mathrm{r}_{2}=1.0 \mu \mathrm{m}$. Thus, it is easy to understand the Rayleigh-like behaviour observed, because only the first oscillations or part of the $\mathrm{Q}_{\text {sca }}$ vs. X curves is been considered in the size distribution, which corresponds to small size parameters.

Our calculations also show how the high-frequency ripples are strongly influenced by the shape. This effect is clearer observed for the refractive index of $1.5+0.001 \mathrm{i}$ with the aggregates of spheres than for the refractive index of $1.62+0.09 \mathrm{i}$ used for the rest of shapes. Changes in the absorption or in the real part of the refractive index produce not only an effect on the interference structure but also on the ripples structure. It takes great values 
of absorption to eliminate the ripples and still greater (about 0.1) to eliminate the interference structure. On the contrary, large values of the real part of the refractive index increase the ripple structure becoming less pronounced the interference structure (see chapter 9 in reference [14]). All these results indicate that if the real and imaginary parts of the refractive index become large at the same time, the effect on the scattering matrix elements for a fixed shape will be the opposite of resembling the Rayleigh features; even stopping the size distribution at $1.0 \mu \mathrm{m}$. We have checked this with calculation using the shape B3 and an equal-size configuration but having spherical monomers instead of cubes and a value of the refractive index of 2.0+4.0i. The optic geometric regime is reached despite we cut the size distribution at $1.0 \mu \mathrm{m}$.

\section{ACKNOWLEDGEMENTS}

We are grateful to B.T. Draine and P.J. Flatau for making their DDA code available.

\section{References}

[1] Muñoz O, Volten H, Haan JF, Vassen W, Hovenier JW. Experimental determination of scattering matrices of randomly oriented fly ash and clay particles at $441.6 \mathrm{~nm}$ and $632.8 \mathrm{~nm}$. J Geophys Res 2001; 106: 833-844.

[2] Kolokolova L, Kimura H, Kiselev N, Rosenbush V. Two different evolutionary types of comets proved by polarimetric and infrared properties of their dust. Astron Astrophys 2007; 463: 1189-1196. 
[3] Kimura H, Kolokolova L, Mann I. Light scattering by cometary dust numerically simulated with aggregate particles consisting of identical spheres. Astron Astrophys 2006; 449:1243-1254.

[4]Nousiainen T. Optical modeling of mineral dust particles: A review. J Quant Spectrosc Radiat Transf 2009;110:1261-1279.

[5] Vilaplana R, Moreno F, Molina A. Study of the sensitivity of sizeaveraged scattering matrix elements of non-spherical particles to changes in shape, porosity and refractive index. J Quant Spectrosc Radiat Transf 2006;100:415-428.

[6] Moreno F, Muñoz O, Guirado D, Vilaplana R. Comet dust as a size distribution of irregularly shaped, compact particles. J Quant Spectrosc Radiat Transf 2007;106:348-359.

[7] Shen Y, Draine BT, Johnson ET. Modeling porous dust grains with ballistic aggregates. II. Light scattering properties. Astron Astrophys 2009;696: 2126-2137.

[8] Draine BT, Flatau PJ. User Guide to the Discrete Dipole Approximation Code DDSCAT 7.1. http://arXiv.org/abs/1002.1505v1 (2010).

[9] Draine BT, Flatau PJ. Discrete-dipole approximation for scattering calculations. J Opt Soc Am A 1994;11:1491-1499.

[10] Zakharova NT, Mishchenko MI. Scattering properties of needlelike and platelike ice spheroids with moderate size parameters. Appl Opt 2000;39:5052-5057. 
[11] Nousiainen T, Vermeulen K. Comparison of measured single-scattering matrix of feldspar particles with T-matrix simulations using spheroids. JQSRT 2003;79-80:1031-1042.

[12] Moreno F, Vilaplana R, Muñoz O, Molina A, Guirado D. The scattering matrix for size distributions of irregular particles: An application to an olivine sample. J Quant Spectrosc Radiat Transf 2006;100:277-287.

[13] Mishchenko MI, Hovenier JW, Lacis AA. Light Scattering by Nonspherical Particles. San Diego: Academic Press 2000; p. 274-307 [Chapter 9].

[14] Mishchenko MI, Travis LD, Lacis AA. Scattering, Absorption and Emission of light by small particle. Cambrigde University Press 2002; p. 238-249 [Chapter 9].

[15] Van Hulst HC. Light scattering by small particles. New York:Dover; 1957. 

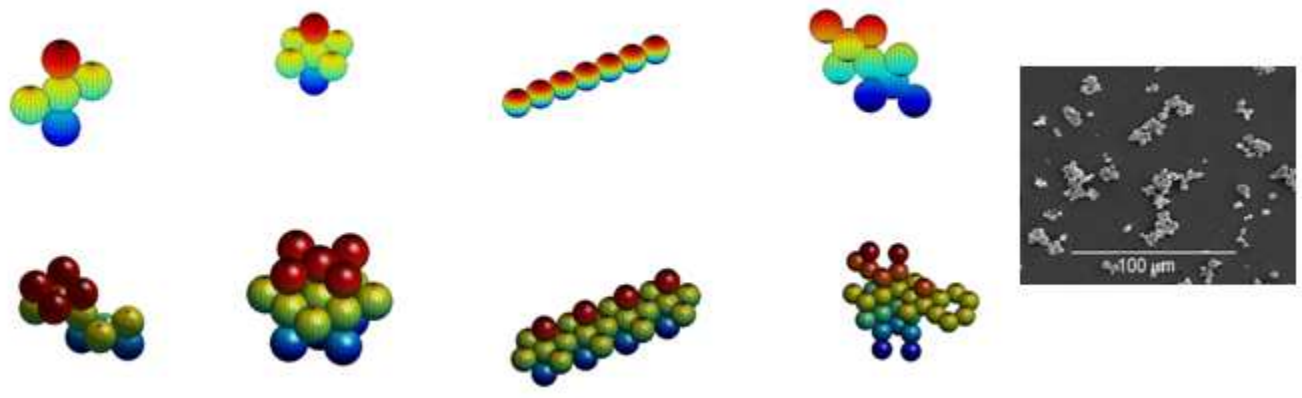

Figure 1: Eight aggregates made of 5, 7, 7in a line, 9, 14, 19, 25 and 36 spheres. 

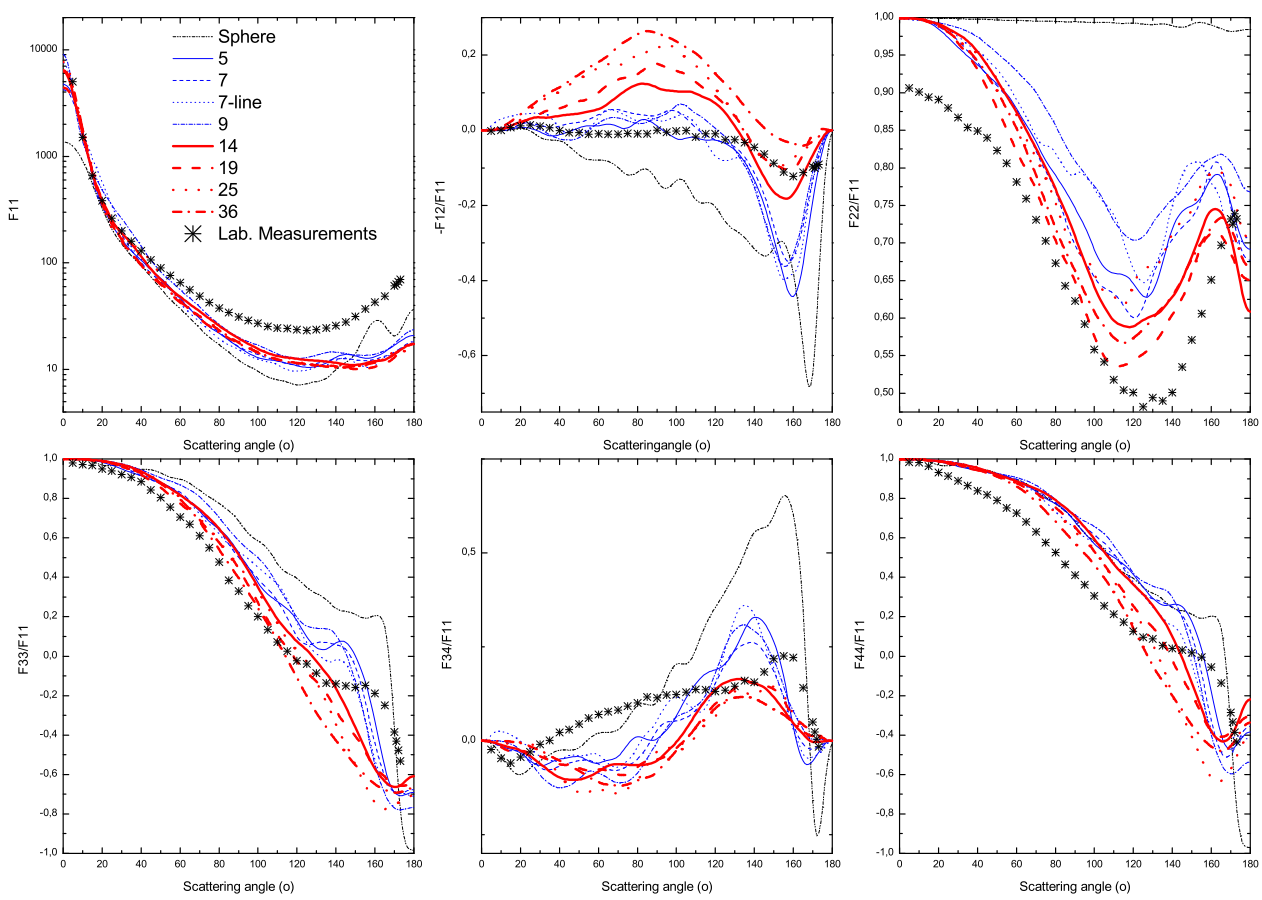

Figure 2: Comparison of laboratory measurements of the single scattering matrix elements of fly ashes with our size averages from 0.1 to $1.0 \mu \mathrm{m}$ for each of the aggregates of spheres (blue and red lines) and for a single sphere (dashed-dot-dot black line). A refractive index of $\mathrm{m}=1.5+0.001 \mathrm{i}$ was used for the calculations along with a wavelength of $0.633 \mu \mathrm{m}$. The elements of the scattering matrix were size-averaged over a power law distribution with an index of -1.8 and 35 equally spaced radii between 0.1 and $1.0 \mu \mathrm{m}$. 

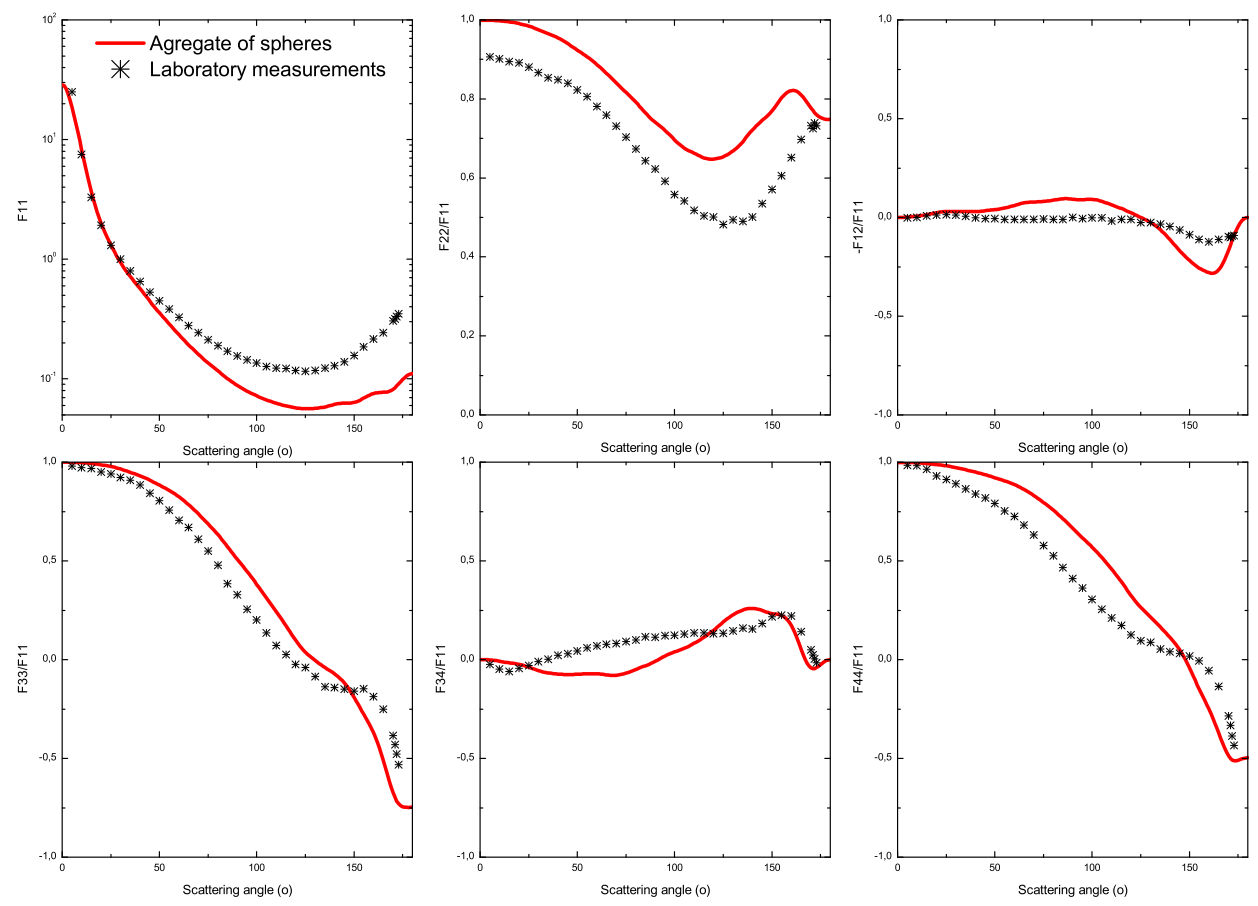

Figure 3: A comparison of laboratory measurements of fly ashes with our size and shape averages from 0.1 to $1.0 \mu \mathrm{m}$, considering the eight aggregates of Fig. 1 and a single sphere, equally weighted. 


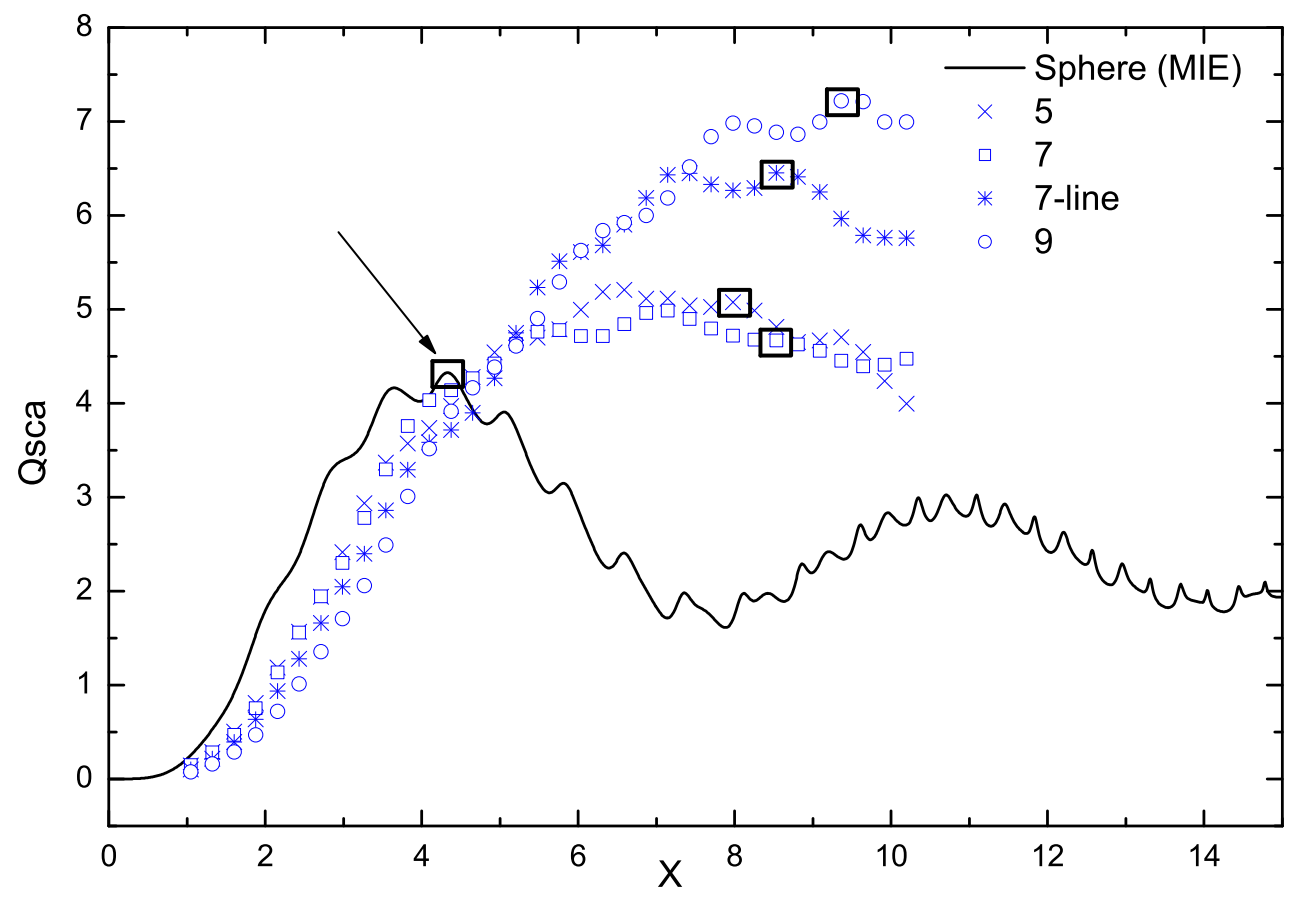

Figure 4: $\mathrm{Q}_{\text {sca }}$ versus $\mathrm{X}$ for the aggregates of Fig. 1 with a number of spheres $\leq 9(5: \times$, 7: $\square, 7$-line: * and 9: $\circ$ ) and a single sphere (Mie: solid line). The squares on the $\mathrm{Q}_{\text {sca }}$ curves of the aggregates with 5, 7, 7-line and 9 spheres are considered in the "same" state of oscillation as that marked by an arrow on the $\mathrm{Q}_{\text {sca }}$ curve of the single sphere (solid line). A refractive index of $\mathrm{m}=1.5+0.001 \mathrm{i}$ was used for the calculations along with a wavelength of $0.633 \mu \mathrm{m}$. 

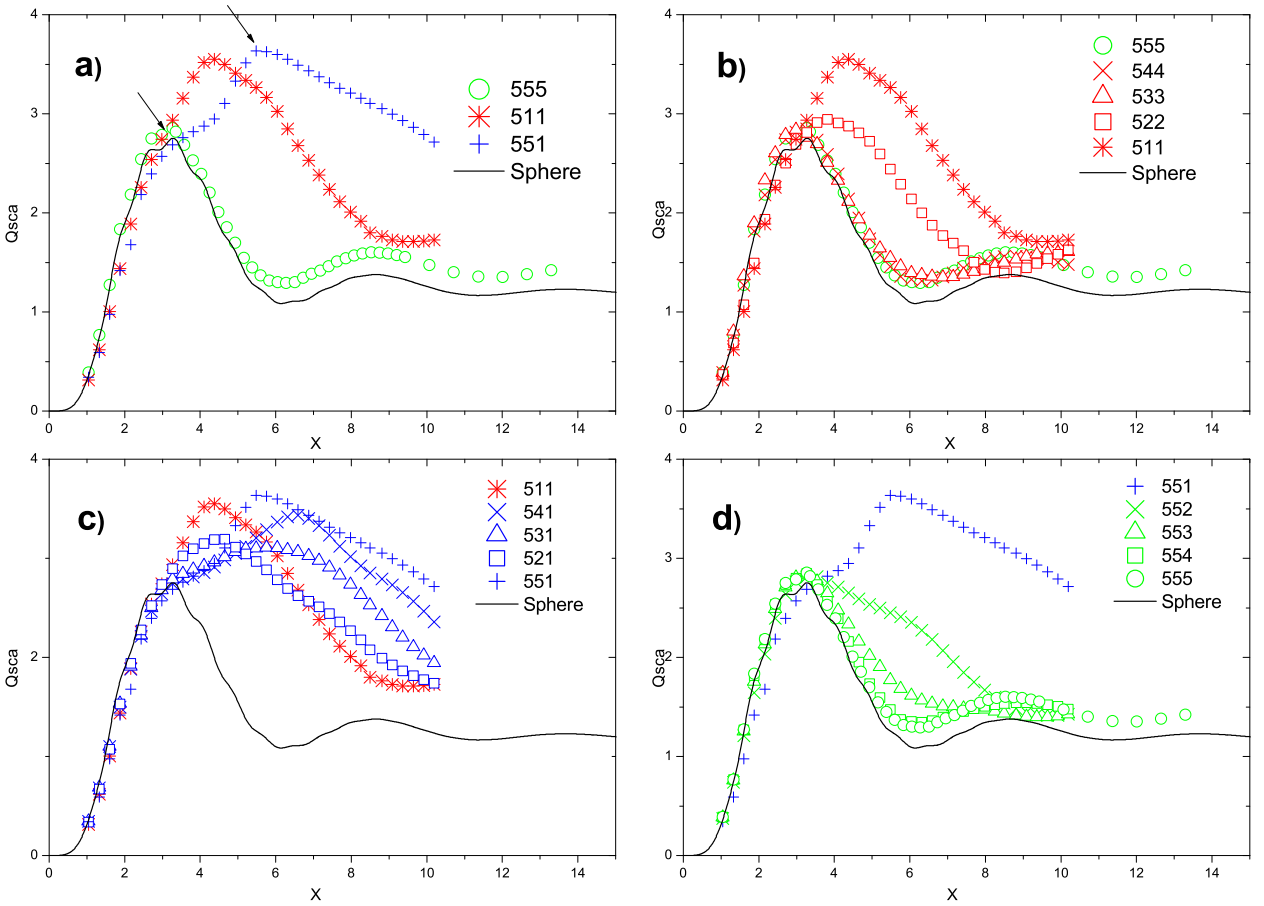

Figure 5: $\mathrm{Q}_{s c a}$ vs. X curves for rectangular prisms with different axial proportions (5:5:5, $5: 4: 4,5: 3: 3,5: 2: 2,5: 1: 1,5: 4: 1,5: 3: 1,5: 2: 1,5: 5: 1,5: 5: 2,5: 5: 3$ and 5:5:4) compared with the results for a sphere. A refractive index of $\mathrm{m}=1.62+0.09 \mathrm{i}$ was used for the calculations along with a wavelength of $0.6 \mu \mathrm{m}$. The elements of the scattering matrix were sizeaveraged over a power law distribution with an index of -1.8 and 35 equally spaced radii between 0.1 and $1.0 \mu \mathrm{m}$. 


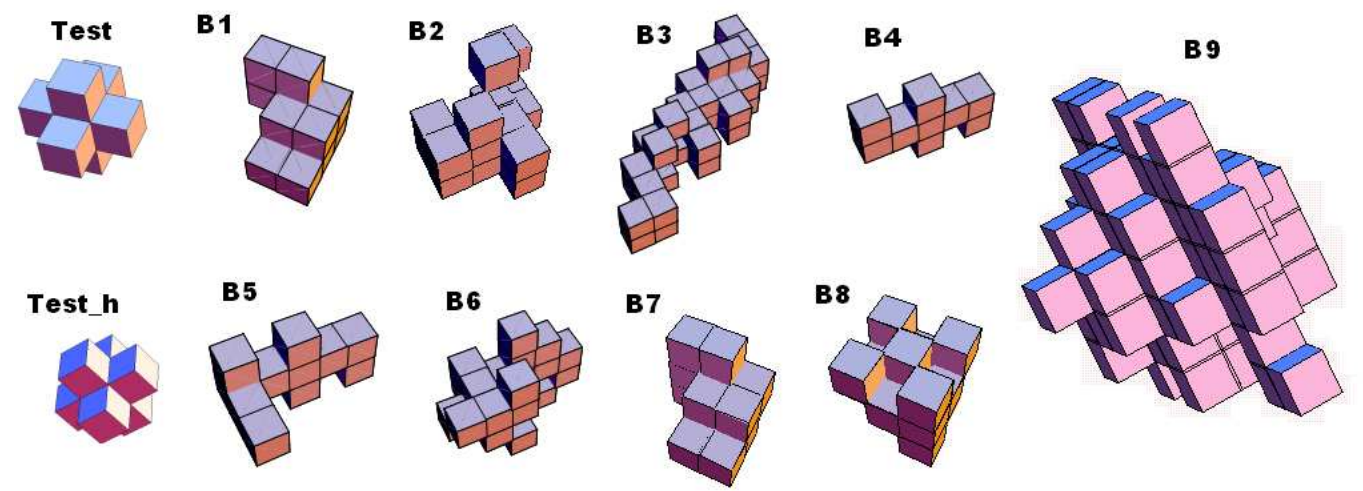

Figure 6: Ten shapes formed by joined cubes: Test, Test_h, B1, B2, B3, B4, B5, B6, B7, B8 and B9 and the labels used to refer them. 

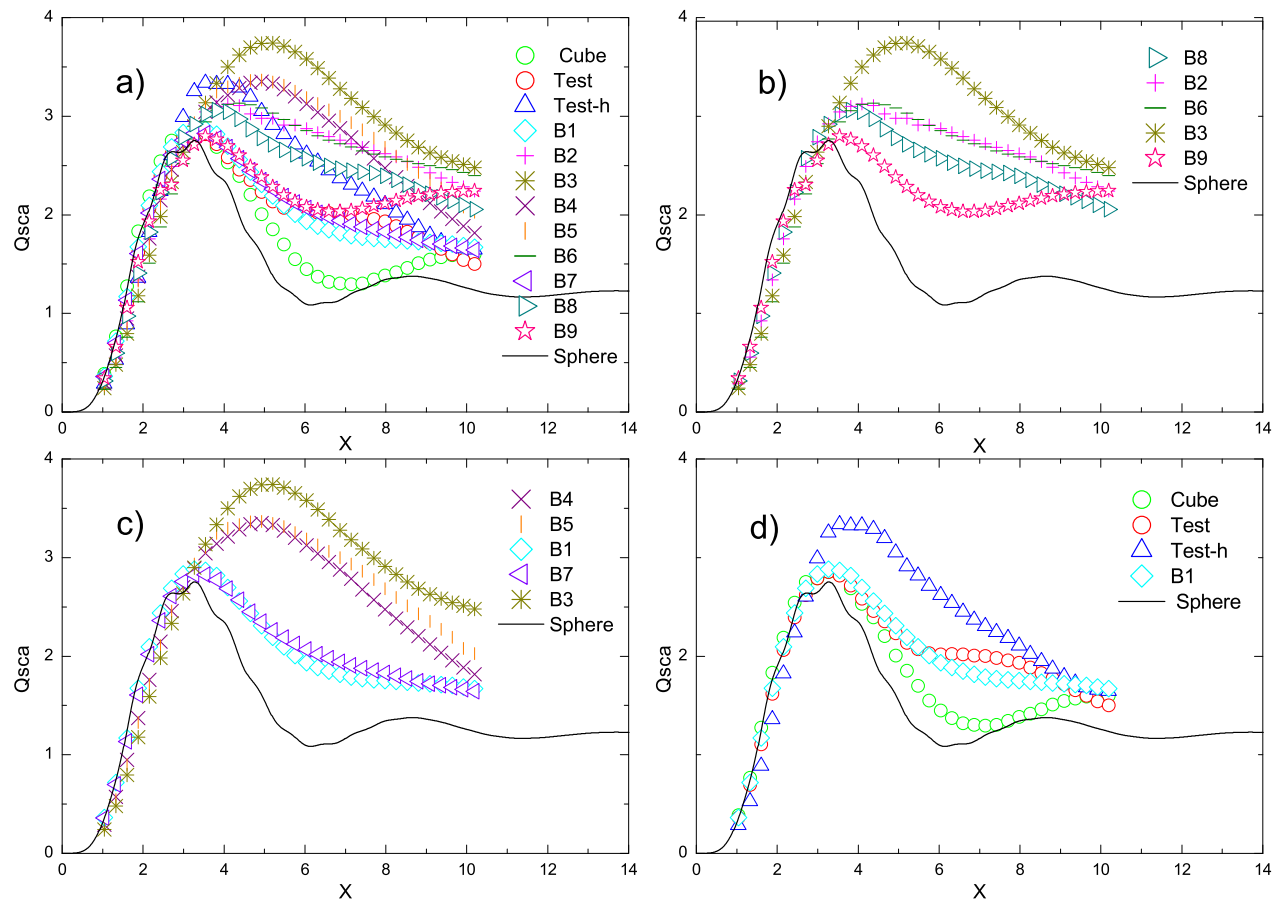

Figure 7: The overlapped curves of the $\mathrm{Q}_{s c a}$ vs. X for different combinations of the shapes in Fig. 6: a) All shapes plus a cube; b) B8, B2, B6, B3 and B9; c) B4, B5, B1, B7 and B3; d) Test, Test-h, B1 plus a cube; and a single sphere. A refractive index of $\mathrm{m}=1.62+0.09 \mathrm{i}$ was used for the calculations along with a wavelength of $0.6 \mu \mathrm{m}$. The elements of the scattering matrix were size-averaged over a power law distribution with an index of -1.8 and 35 equally spaced radii between 0.1 and $1.0 \mu \mathrm{m}$. 

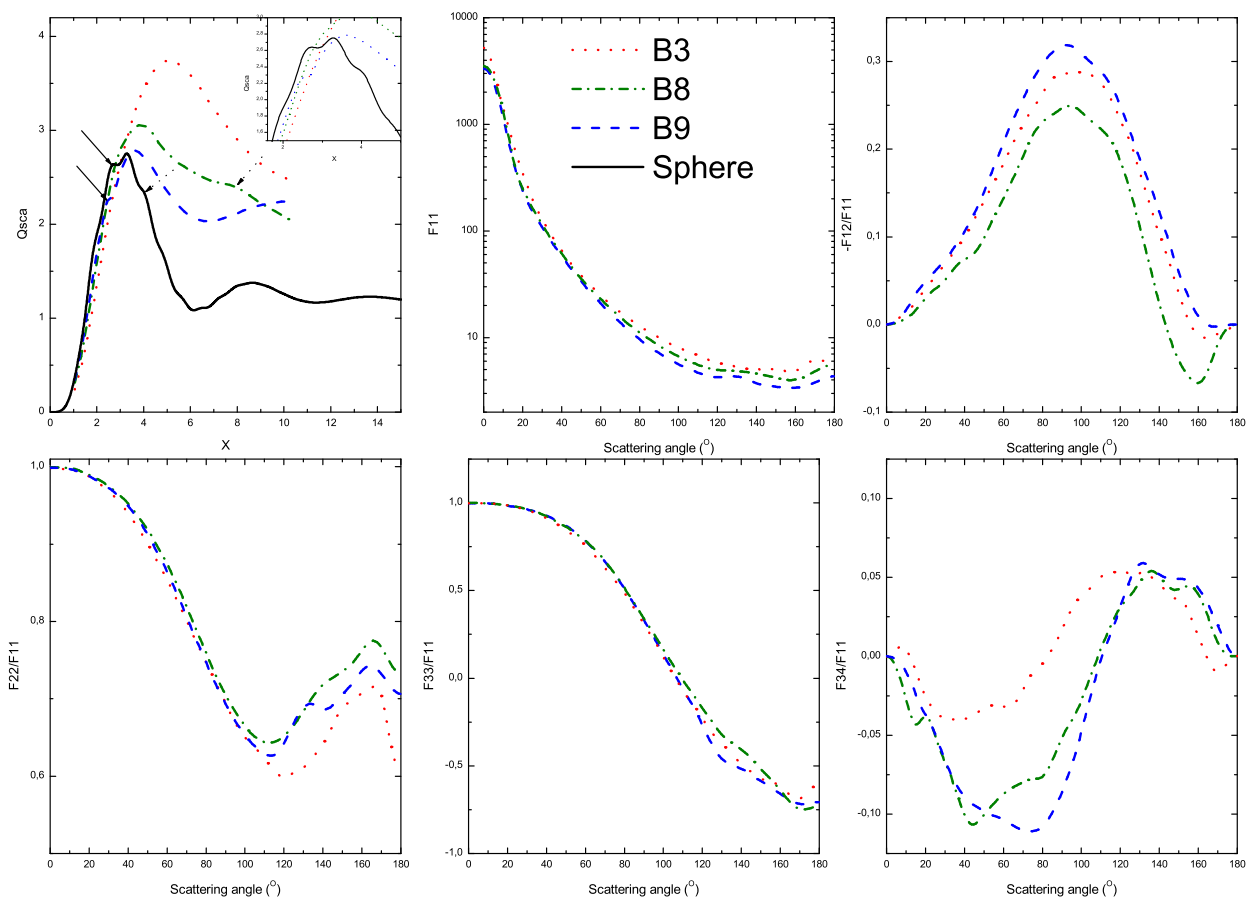

Figure 8: $\mathrm{Q}_{\text {sca }}$ vs. $\mathrm{X}$ curves and corresponding size-averaged scattering matrix elements as functions of the scattering angle (excluding $\mathrm{F}_{44} / \mathrm{F}_{11}$ ) for shapes B3, B8, and B9. A refractive index of $\mathrm{m}=1.62+0.09 \mathrm{i}$ was used for the calculations along with a wavelength of $0.6 \mu \mathrm{m}$. The elements of the scattering matrix were size-averaged over a power law distribution with an index of -1.8 and 35 equally spaced radii between 0.1 and $1.0 \mu \mathrm{m}$. 

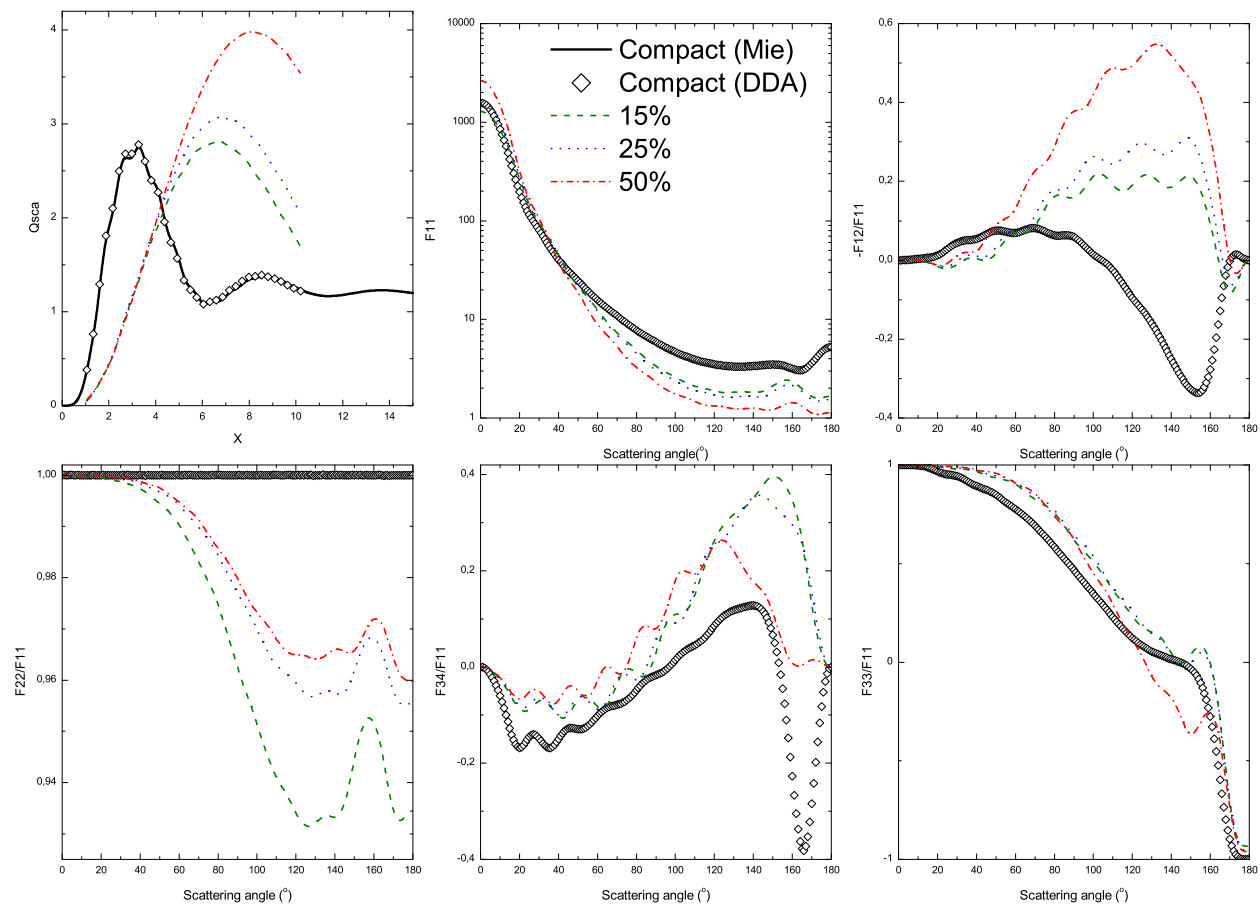

Figure 9: $\mathrm{Q}_{s c a}$ vs. $\mathrm{X}$ and the corresponding size-averaged scattering matrix elements (excluding the $\mathrm{F}_{44} / \mathrm{F}_{11}$ ) as functions of the scattering angle for a single sphere and the sphere with the fluffiness degrees of $15 \%, 25 \%$ and $50 \%$. A refractive index of $\mathrm{m}=$ $1.62+0.09 \mathrm{i}$ was used for the calculations along with a wavelength of $0.6 \mu \mathrm{m}$. The elements of the scattering matrix were size-averaged over a power law distribution with an index of -1.8 and 35 equally spaced radii between 0.1 and $1.0 \mu \mathrm{m}$. 

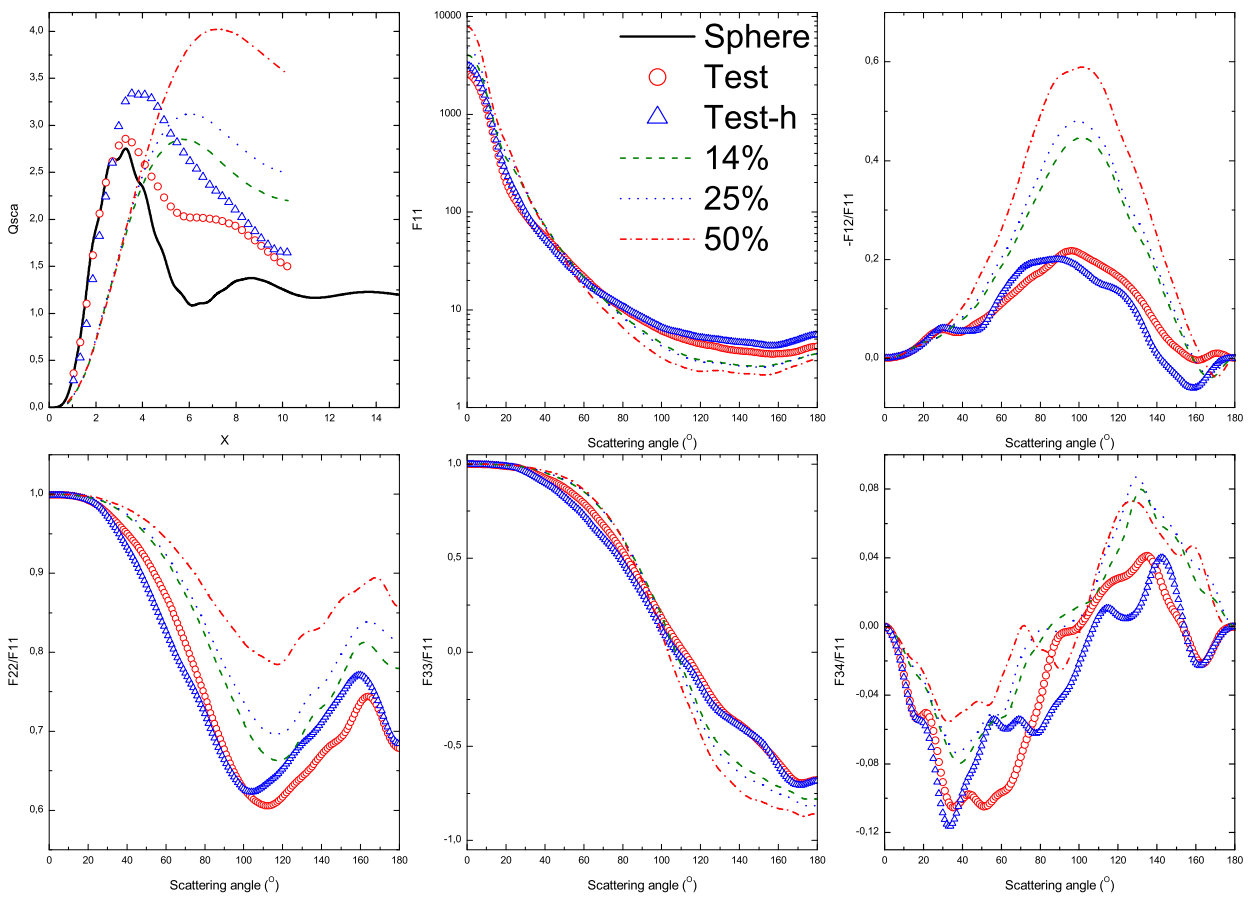

Figure 10: $\mathrm{Q}_{s c a}$ vs. $\mathrm{X}$ and the corresponding size-averaged scattering matrix elements (excluding the $\mathrm{F}_{44} / \mathrm{F}_{11}$ ) as functions of the scattering angle for shapes Test, Test-h and Test with the fluffiness degrees of $14 \%, 25 \%$ and $50 \%$. A refractive index of $\mathrm{m}=1.62+0.09 \mathrm{i}$ was used for the calculations along with a wavelength of $0.6 \mu \mathrm{m}$. The elements of the scattering matrix were size-averaged over a power law distribution with an index of -1.8 and 35 equally spaced radii between 0.1 and $1.0 \mu \mathrm{m}$. 

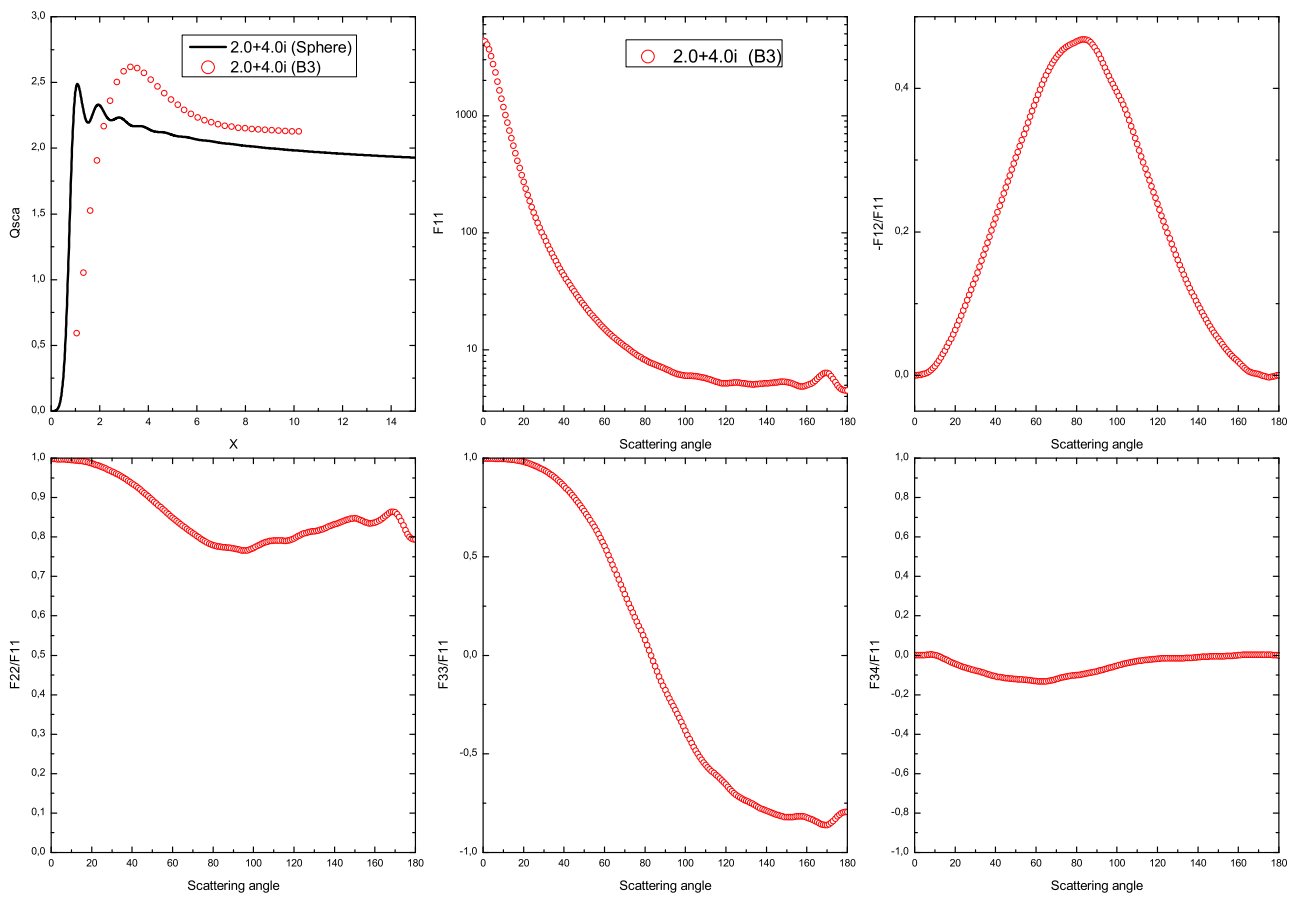

Figure 11: $\mathrm{Q}_{s c a}$ vs. $\mathrm{X}$ and the corresponding size-averaged scattering matrix elements (excluding the $\mathrm{F}_{44} / \mathrm{F}_{11}$ ) as functions of the scattering angle for shape B3. A refractive index of $\mathrm{m}=2.0+4.0 \mathrm{i}$ was used for the calculations. The elements of the scattering matrix were size-averaged over a power law distribution with an index of -1.8 and 35 equally spaced radii between 0.1 and $1.0 \mu \mathrm{m}$. 